\title{
INTERACTIONS OF WAVES WITH AN ARRAY OF TANDEM PLACED BOTTOM-MOUNTED CYLINDERS
}

Baris Barlas

Department of Naval Architecture \& Marine Engineering, Istanbul Technical University, Istanbul, Turkey, barlas@itu.edu.tr

Follow this and additional works at: https://jmstt.ntou.edu.tw/journal

Part of the Engineering Commons

\section{Recommended Citation}

Barlas, Baris (2012) "INTERACTIONS OF WAVES WITH AN ARRAY OF TANDEM PLACED BOTTOM-MOUNTED CYLINDERS," Journal of Marine Science and Technology. Vol. 20: Iss. 1, Article 13.

DOI: $10.51400 / 2709-6998.2428$

Available at: https://jmstt.ntou.edu.tw/journal/vol20/iss1/13

This Research Article is brought to you for free and open access by Journal of Marine Science and Technology. It has been accepted for inclusion in Journal of Marine Science and Technology by an authorized editor of Journal of Marine Science and Technology. 
INTERACTIONS OF WAVES WITH AN ARRAY OF TANDEM PLACED BOTTOMMOUNTED CYLINDERS

Acknowledgements

The author would like to thank his colleague Dr. Serdar Beji with his precious discussions. 


\section{INTERACTIONS OF WAVES WITH AN ARRAY OF TANDEM PLACED BOTTOM-MOUNTED CYLINDERS}

\begin{abstract}
Diffraction of water waves with an array of bottommounted cylinders of circular cross section is studied. A vertically-integrated nonlinear dispersive wave model with inclusion of the wave breaking effect in a semi-empirical manner, in nonorthogonal curvilinear coordinate system is used for simulating linear and nonlinear wave forces on bottom mounted tandem placed circular cylinders. The wave model is in terms of the contravariant velocities. The mesh required is generated based on staggered grids on the freesurface. The matrix equation of the finite difference method is solved through an iterative approach at each time step. For outgoing waves Sommerfeld's first order radiation condition is used. The free-surface elevation and hydrodynamic forces on each cylinder are calculated to illustrate the evolution of water waves and their interactions with cylinder arrays. Comparisons of linear and nonlinear force coefficients and relevant discussion for two and three cylinder arrays are presented in closing.
\end{abstract}

\section{INTRODUCTION}

The study of hydrodynamic interaction among an array of bottom-mounted cylinders is an important issue and has received significant interest in recent years. This arrangement has a wide range of practical and engineering applications, such as oil platforms, TLPs, jetty legs and bridges. The hydrodynamic interaction among neighboring components of bottom-mounted cylinders may notably affect the wave field, wave loads and wave run-up on the individual structures, which differ significantly from what they would experience in standing alone case. As the relative size of structures in-

Paper submitted 08/24/10; revised 10/18/11; accepted 11/01/11. Author for correspondence: Baris Barlas (e-mail: barlas@itu.edu.tr).

Department of Naval Architecture \& Marine Engineering, Istanbul Technical University, Istanbul, Turkey. creases, the scattering of nonlinear waves becomes more important for the determination of wave run-up and wave forces on those structures. The effect of a given incident wave on an individual structure is to produce a scattered wave which in turn is scattered by neighboring components. If the cylinder diameter is sufficiently large compared with the wavelength and wave amplitude, then the scattering of nonlinear waves and the diffraction effects become more important.

The computation of the wave diffraction due to an array of cylinders can be achieved either in the frequency domain or in the time domain. One of the advantages of the time domain method over the frequency domain method is that it can capture the transient effects.

Early works on the wave diffraction problem on vertical circular cylinders were associated with the estimation of wave forces, that can be calculated from the first order potential [9, 15]. Su and Pan [18] investigated the cnoidal wave forces on cylinder arrays. The scattering of water waves incident upon a group of four cylinders situated at the vertices of a square was solved by Linton and Evans [11].

Works mostly concentrated on the analysis of the wave diffraction problem on vertical circular cylinders $[1,8,9,11$, $13,15]$.

For the nonlinear wave interactions with structures in shallow water, Wang et al. [24] and Wang and Jiang [22] presented a Boussinesq model in curvilinear coordinate system. Wang and Jiang [22] investigated solitary wave interactions with an array of two vertical cylinders. Carstens and Sayer [6] used linear potential theory to investigate the hydrodynamic interactions between two vertical cylinders in harmonic flow. Maniar and Newman [12] studied the forces on linear arrays of bottom mounted circular cylinders aligned along an axis. Wang and Ren studied the scattering of cnoidal waves by cylinder arrays by using the generalized Boussinesq equations [23]. Utsunomiya and Eatock Taylor examined the trapped modes around a row of bottom-mounted vertical circular cylinders in a channel [19]. Williams and Li [25] investigates the interaction of water waves with arrays of bottom mounted circular cylinders under the assumptions of potential flow and linear wave theory. The important aspect of this work is the porous sidewall of each cylinder. Ohl et al. [17] investigated the diffraction of regular waves by arrays of 


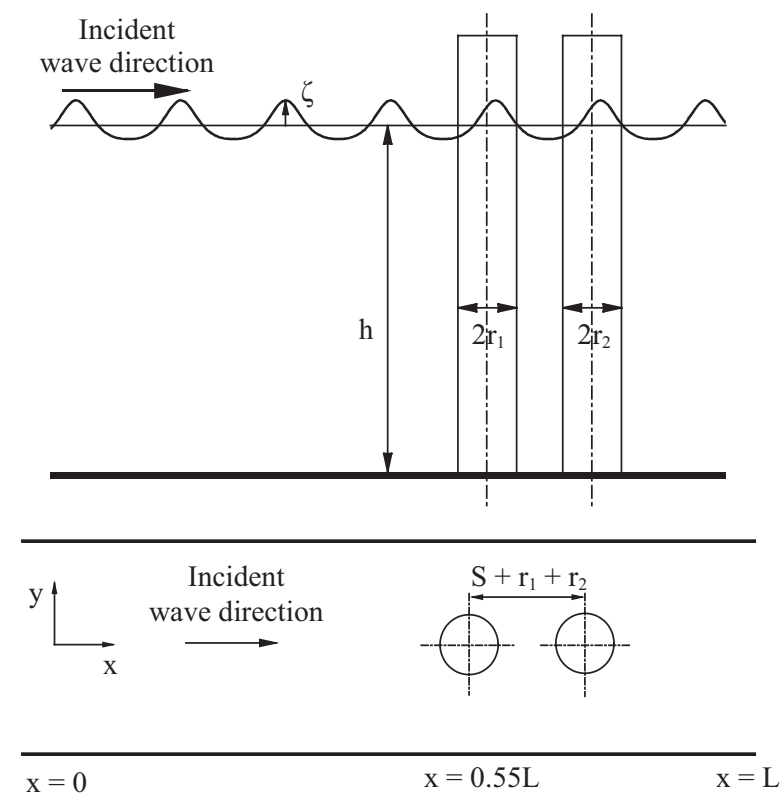

Fig. 1. Schematic diagram of the problem for tandem placed cylinder array used in the simulations.

vertical bottom-mounted circular cylinders using theoretical, computational, and experimental methods.

Yilmaz [26] studied the diffraction of water waves by an array of vertical cylinders of circular cross section. The body boundary condition was satisfied for each cylinder considering the scattered wave field from other cylinders in an iterative way in order to take account for the first order interaction among the cylinders. Walker and Eatock Taylor [20] investigated the diffraction of regular waves by linear arrays of vertical bottom mounted circular cylinders under the assumption of linear theory. Mavrakos and Chatjigeorgiou [14] studied the second-order diffraction potential around a bottom-seated compound cylinder. The solution method was based on a semi-analytical formulation for the double-frequency diffraction potentials. Wang and $\mathrm{Wu}$ [21] employed a time domain method to analyze interactions of water waves by an array of two cylinders, four cylinders, ten cylinders and two arrays of eight cylinders.

The present work investigates the scattering of water waves by an array of cylinders. For this problem a verticallyintegrated nonlinear dispersive wave model together with a semi-empirical wave breaking effect is used to compute the wave field and hydrodynamic wave loads. The wave equations in nonorthogonal curvilinear coordinates are numerically solved using finite difference approximations. Only half of the circular cylinders about the symmetry axis is placed inside the physical domain. A series of numerical simulations were carried out for linear sinusoidal and nonlinear cnoidal waves on tandem placed circular cylinders, having different spacing between cylinders and $\mathrm{kr}$ values to examine the effects of cylinder configuration on the wave profiles and wave forces.
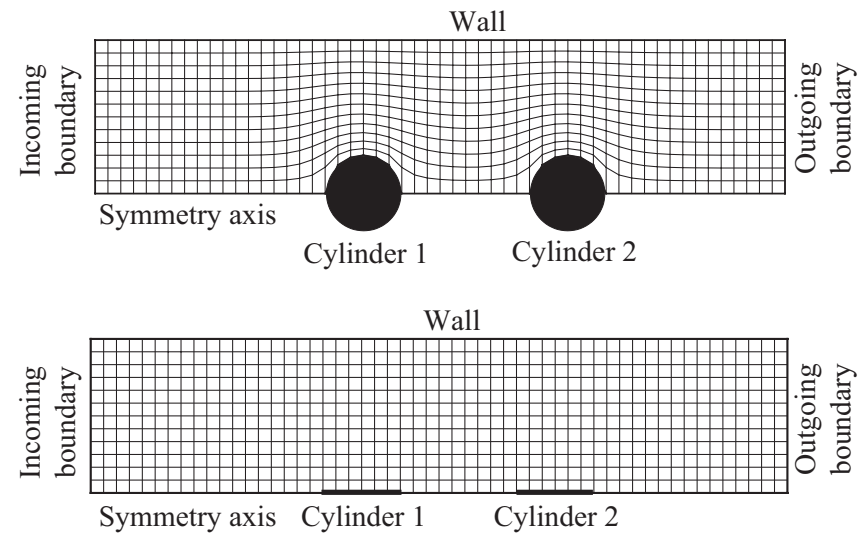

Fig. 2. A typical grid arrangement in the physical domain $(x, y)$ and computational domain $(\xi, \eta)$ for tandem placed cylinder array.

\section{DESCRIPTION OF THE PROBLEM}

The present work considers the wave diffraction problem by an array of bottom-mounted piles of circular cross section cylinders. Fig. 1 describes the side and plan views of the problem with coordinate system used. The $x$ and $y$ are measured horizontally and $\mathrm{z}$ measured vertically upwards from the still water level. The incident waves are traveling from the left at $x=0$ along the $y$-axis. The first cylinder is placed at $0.55 \mathrm{~L}$, to the right of the mid-domain, to delay the contaminating effects of the reflected waves traveling back to the incoming boundary. $S$ is the space between cylinders. At the end of the domain $(x=L)$ an appropriate radiation condition is utilized.

The numerical solution is carried out using a boundaryfitted nonorthogonal curvilinear coordinate system. Fig. 2 shows a typical grid arrangement for the physical domain in the $(x, y)$ coordinates and its counterpart in the computational domain in the $(\xi, \eta)$ coordinates. Due to the symmetry of the problem only half of the domain is discretized as given in Barlas and Beji [2].

\section{FORMULATION OF THE PROBLEM}

\section{Wave Model}

The wave model used in this work is the one-component form of the fully-dispersive vertically-integrated nonlinear model of Nadaoka et al. [16] as expressed in nonorthogonal curvilinear coordinates by Beji and Barlas [3] with newly insertion of a semi-empirical wave breaking effect. The momentum equations in the $\xi$ - and $\eta$-directions can be obtained by combining the $x$ - and $y$-momentum equations:

$$
\begin{aligned}
& \xi_{x}(x \text {-momentum })+\xi_{y}(y \text {-momentum })= \\
& \eta_{x}(x \text {-momentum })+\eta_{y}(y \text {-momentum })=0
\end{aligned}
$$

In the curvilinear coordinate system the continuity, $\xi$-momentum and $\eta$-momentum equations can be written as, 


$$
\begin{gathered}
\zeta_{\tau}+J\left[\left(\frac{C_{p}^{2}}{g}+\zeta\right) U^{*}\right]_{\xi}+J\left[\left(\frac{C_{p}^{2}}{g}+\zeta\right) V^{*}\right]_{\eta}=0 \\
r J U_{\tau}^{*}+\xi_{*}^{2} Q_{\xi}+\xi_{*} \eta_{*} Q_{\eta}= \\
\frac{\xi_{*}^{2}}{\omega^{2} C_{p}^{2}}\left[C_{*}\left(U_{\xi}^{*}+V_{\eta}^{*}\right)\right]_{\xi \tau}+\frac{\xi_{*} \eta_{*}}{\omega^{2} C_{p}^{2}}\left[C_{*}\left(U_{\xi}^{*}+V_{\eta}^{*}\right)\right]_{\eta \tau}+\alpha B \\
r J V_{\tau}^{*}+\eta_{*}^{2} Q_{\eta}+\xi_{*} \eta_{*} Q_{\xi}= \\
\frac{\eta_{*}^{2}}{\omega^{2} C_{p}^{2}}\left[C_{*}\left(U_{\xi}^{*}+V_{\eta}^{*}\right)\right]_{\eta \tau}+\frac{\xi_{*} \eta_{*}}{\omega^{2} C_{p}^{2}}\left[C_{*}\left(U_{\xi}^{*}+V_{\eta}^{*}\right)\right]_{\xi \tau}+\alpha B
\end{gathered}
$$

where

$$
\begin{gathered}
Q=g \zeta+\frac{1}{2}\left(1-3 \omega^{2} C_{p}^{2} / g^{2}\right)\left(\eta_{*}^{2} U^{* 2}+\xi_{*}^{2} V^{* 2}-2 \xi_{*} \eta_{*} U^{*} V^{*}\right) \\
B=\left(\xi_{x x}+\xi_{y y}\right) \frac{\partial U^{*}}{\partial \xi}+\left(\eta_{x x}+\eta_{y y}\right) \frac{\partial U^{*}}{\partial \eta}+\xi_{*}^{2} \frac{\partial^{2} U^{*}}{\partial \xi^{2}} \\
+\eta_{*}^{2} \frac{\partial^{2} U^{*}}{\partial \eta^{2}}+2 \xi_{*} \eta_{*} \frac{\partial^{2} U^{*}}{\partial \xi \partial \eta} \\
\xi_{*}^{2}=\xi_{x}^{2}+\xi_{y}^{2}, \eta_{*}^{2}=\eta_{x}^{2}+\eta_{y}^{2}, \\
\xi_{*} \eta_{*}=\xi_{x} \eta_{x}+\xi_{y} \eta_{y}, C_{*}=C_{p}^{4}(1-r) J \\
J=\frac{1}{x_{\xi} y_{\eta}-x_{\eta} y_{\xi}} \\
\alpha=\frac{1}{\mu} e^{|\xi|}
\end{gathered}
$$

where $r=C_{g} / C_{p}, g$ is the gravitational acceleration, $\zeta$ is the free surface elevation. $C_{p}$, and $C_{g}$, denote respectively the phase and group velocities, computed according to the linear theory for a prescribed dominant frequency $\omega$ and a given local depth $\mathrm{h}$. The term $\alpha B$ is the emprical breaking term. $\mu$ is the eddy viscosity, which is taken $10^{-4}$. $J$ is the Jacobian of the transformation. $\xi_{x}, \xi_{y}, \eta_{x}, \eta_{y}$ are the metrics of the grid system. Subscripts indicate partial differentiation with respect to the indicated variable in the curvilinear coordinates. $U^{*}$ and $V^{*}$ are defined as,

$$
\begin{gathered}
U^{*}=u y_{\eta}-v x_{\eta}=\frac{u \xi_{x}+v \xi_{y}}{J} \\
V^{*}=-u y_{\xi}-v x_{\xi}=\frac{u \eta_{x}+v \eta_{y}}{J}
\end{gathered}
$$

\section{Breaking of Waves}

The one-component form of the fully-dispersive vertically-integrated nonlinear model of Nadaoka et al. [16], like most of the other wave models in the literature, cannot model the wave overturning and breaking. A wave overturns and then breaks at the wave crest when the skewness, a measure of crest-trough shape, exceeds a critical value and the orbital speed of the water particles at the wave crest becomes higher than the wave celerity. An asymmetric wave profile starts to occur, then once the wave overturns and breaks, it continues to break until it reaches at some smaller stable level at which it reforms itself [7].

The proposed modified wave model predicts dissipation of energy due to breaking in an emprical manner as a numerical dissipation which is related to the mean velocity gradient that becomes large when the skewness becomes large. Thus an energy dissipation model can be built into the equation by the addition of a wave breaking damping term $\alpha B$, to simulate the wave breaking effect. The magnitude of the coefficient $\alpha$ is infinitesimally small when breaking does not occur, but grows to a significant value when skewness becomes large and breaking starts. Then the top of the wave crest was peeled off by using the damping term. The wave model prevents the skewness from exceeding a critical value. The evaluation of the skewness at each grid point can be computed from elevation at chosen neighboring grid locations. Wave heights, hence, the skewness near cylinders grow to be very large.

\section{Boundary Conditions}

Three different boundary conditions are used: incoming boundary condition, wall and outgoing boundary conditions. At the incoming boundary the incident displacement $\zeta$ is specified as either linear sinusoidal or nonlinear cnoidal wave forms. For sinusoidal waves the corresponding contravariant linear velocity is obtained from the linearized continuity equation as

$$
U^{*}=\frac{\zeta C g}{J\left(C_{p}^{2}\right)}
$$

where $C$ being the phase velocity of the incident wave and the phase velocity $C_{p}$ is taken equal to $C$ for linear simulations. For cnoidal waves the nonlinear velocity is obtained from the full form (nonlinear) of the continuity equation as

$$
U^{*}=\frac{\zeta C}{J\left(\frac{C_{p}^{2}}{g}+\zeta\right)}
$$

where $C$ denoting the cnoidal wave celerity. The wave model is in terms of the contravariant velocities therefore the wall condition is satisfied accurately and easily; it may be specified by taking the contravariant velocity component normal to the wall equal to zero. At the outgoing boundary the well known 


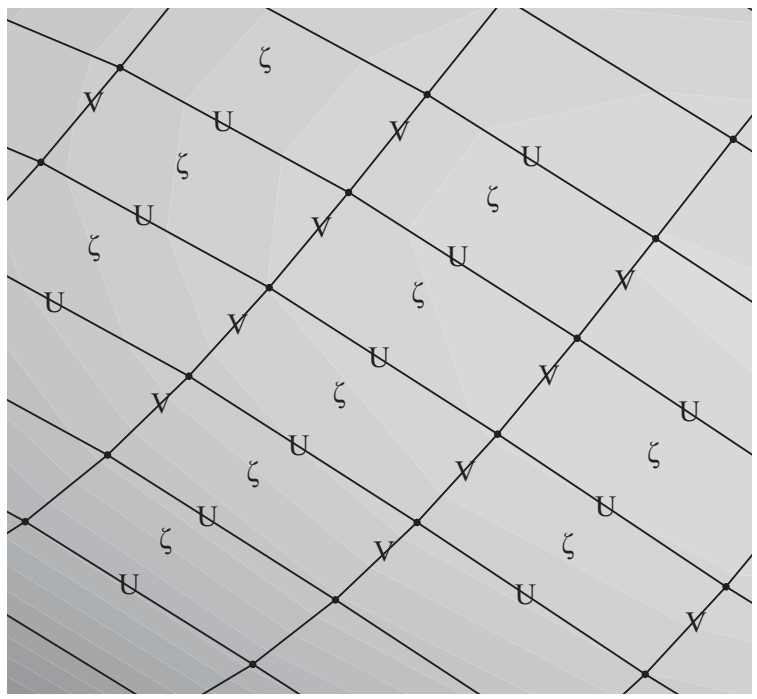

Fig. 3. Staggered grid orientation for the variables on the free-surface.

Sommerfeld's radiation condition is employed. For right going waves, Sommerfeld's equation in curvilinear coordinates in the positive $\xi$-direction can be given by

$$
\begin{gathered}
\left(\xi_{x} \eta_{y}-\xi_{y} \eta_{x}\right) U_{\tau}^{*}+C\left[\left(\xi_{x}^{2} \eta_{y}-\xi_{y}^{2} \eta_{x}\right) U_{\xi}^{*}+\eta_{x} \eta_{y}\left(\xi_{x}-\xi_{y}\right) U_{\eta}^{*}\right] \\
+C \xi_{x} \xi_{y}\left[\left(\xi_{y}-\xi_{x}\right) V_{\xi}^{*}+\left(\eta_{y}-\eta_{x}\right) V_{\eta}^{*}\right]
\end{gathered}
$$

\section{Force Computation}

The wave-induced hydrodynamic force acting on each member of cylinder array is calculated by integrating the pressure distribution on the cylindrical surface. The force in terms of the contravariant velocities is computed using

$$
\begin{aligned}
\bar{F}_{x}= & \frac{F_{x}}{\rho g h H r(\tanh k h / k h)}=\frac{1}{g k^{2} H} \int_{0}^{2 \pi} J\left(U_{\xi}^{*}+V_{\eta}^{*}\right)_{\tau} \cos \theta d \theta \\
& -\frac{k h}{g H \sinh 2 k h} \int_{0}^{2 \pi}\left(\eta_{x}^{2}+\eta_{y}^{2}\right) U^{* 2} \cos \theta d \theta
\end{aligned}
$$

as given in Barlas and Beji [2].

\section{NUMERICAL METHOD}

The numerical procedure used here is the finite difference method, based on the method established by Beji and Barlas [3]. The staggered grid scheme given in Fig. 3 is utilized on the free surface. Staggered grid arrangement has important advantages for depth integrated wave computations. The time derivatives are centered at the mid-time level $\tau+\Delta \tau / 2, \tau$ being the current time and $\Delta \tau$ the time step. All the space derivatives are of second order. The wave propagation direction is taken along the positive $\xi$ - axis; therefore, $\xi$ - momentum equation is solved first for $U^{*}$ then $\eta$ - momentum equation is solved later for $V^{*}$ implicitly. The free-surface elevation is then calculated explicitly from the continuity equation. In the computational domain the space distance between the grids are taken unity, i.e. $\Delta \xi=\Delta \eta=1$. Since the computations involve certain approximations, an iterative procedure is needed. The wave Courant number in the $\mathrm{x}$-axis direction $C r=C \Delta \tau / \Delta x$ should be close to 1 for better results. The iteration criterion is $10^{-5}$ : i.e. the relative error between two iteratively computed values of the variables at each grid point must be less than the specified criterion. The computations is made on a Pentium 4 PC with $3.4 \mathrm{GHz}$ Intel CPU, windows Vista system and Fortran Power Station 4.0 complier.

\section{NUMERICAL RESULTS AND DISCUSSION}

For linear waves a sinusoidal wave form is specified as the incident wave. The water depth is $h=1 \mathrm{~m}$, the wavelength $\lambda$ is $2 \pi$ and accordingly period is $T=2.3 \mathrm{~s}$ for all linear simulations. For nonlinear waves, in shallow water, the solution of the Korteweg and de Vries equation is the cnoidal waves [10]. For simulating the nonlinear wave propagation and wave interactions with tandem placed vertical cylinder array, the cnoidal waves are used as incident waves. Using a series of the Jacobi elliptic functions with the selection of two parameters, wave steepness $\varepsilon$ and elliptical modulus $\mathrm{m}$, the physical wave quantities are calculated. For nonlinear case the cylinder radius is computed from $r=(k r)(\lambda / 2 \pi)$. The water depth is $h=0.25 \mathrm{~m}$. The wavelength $\lambda$ is computed from $\lambda=4 h K(m) \sqrt{m / 3 \varepsilon}$. The nondimensional parameter $k r$, which shows the degree of diffraction, is varied between 0.5 and 2.5 for both linear and nonlinear simulations.

An important feature of the interaction of waves about circular cylinders is the ocurance of vortex shedding at the well known Keulegan-Carpenter numbers above 3 [5]. The Keulegan-Carpenter number can be given as,

$$
K C=\frac{U T}{D}=\frac{\varepsilon \lambda}{D}
$$

where $U$ is the partical velocity, T is the wave period, $D$ is the diameter of cylinder, $\varepsilon$ is the wave steepness, and $\lambda$ is the wave length. In the computations the maximum value of $K C$ number is about 3 , therefore no vortex shedding is expected.

\section{Two Cylinder Array}

The two cylinder array configuration is the simplest multiple cylinder case. The two cylinders arranged in tandem parallel to the wave direction. The $x$-direction grid size $\Delta x$ ranges from $\lambda / 30$ to $\lambda / 75$ and the $y$-direction grid size $\Delta y$ ranges from $\lambda / 20$ to $\lambda / 50$. The incident wave amplitude is $a_{0}=1$, the number of grid points in $x$ - and $y$-directions are 615 and 60 , respectively. The time step $\Delta \tau=0.1(=T / 40)$ s. Fig. 4 shows the three dimensional perspective view and Fig. 5 shows the contour plots of a fully developed sinusoidal wave field in 


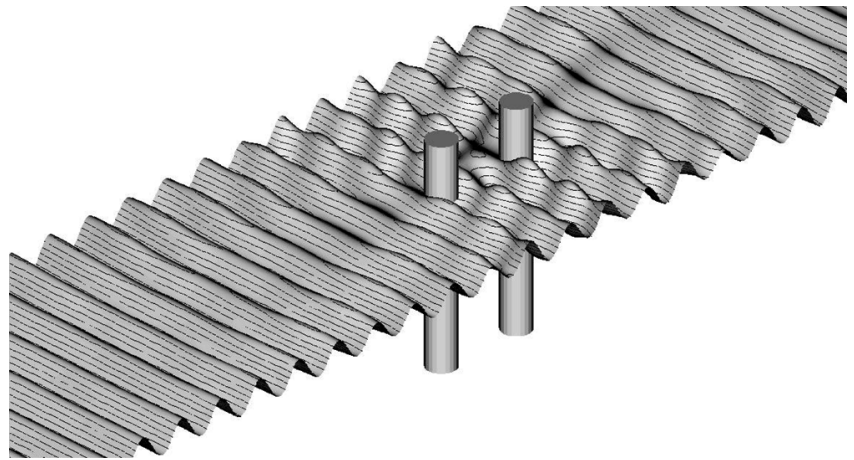

Fig. 4. Perspective view of a fully developed wave field at $t=75 T$ in presence of two circular cylinders for $k r=2.0, S / r=4, \lambda=2 \pi$.

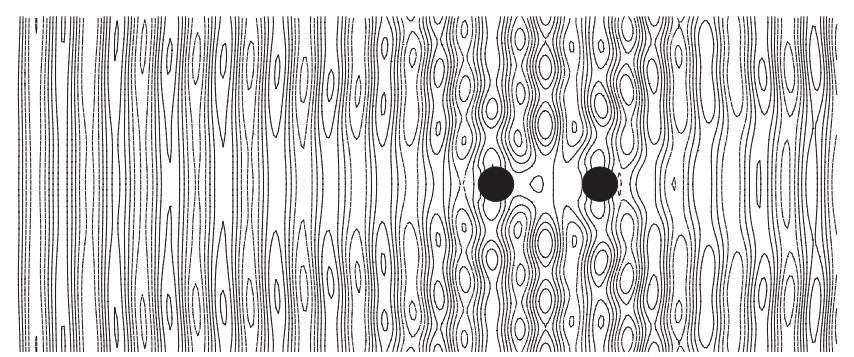

Fig. 5. Contours of a fully developed sinusoidal wave field at $t=75 T$ in presence of two circular cylinders for $k r=2.0, S / r=4, \lambda=2 \pi$.

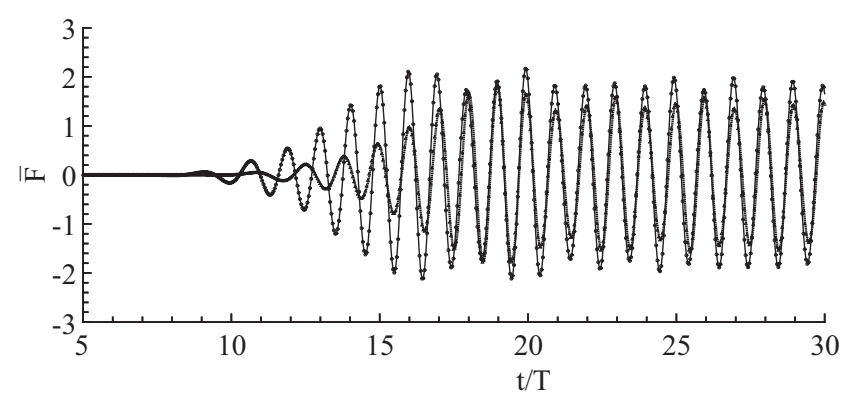

Fig. 6. The computed nondimensional linear wave force on tandem placed circular cylinders for a duration of 25 periods between $t=$ $5 T$ and $t=30 T$ for $k r=2.0, S / r=4, \lambda=2 \pi$.

presence of the two tandem placed cylinder array for $k r=2$, $S / r=4$, after 75 wave periods elapsed from the start.

Fig. 6 depicts the time variation of the computed nondimensional linear wave force on the frontal and rear cylinders for a duration of 25 periods between $t=5 \mathrm{~T}$ and $t=30 \mathrm{~T}$ for $k r=2, S / r=4, \lambda=2 \pi$. The solid line represents wave forces on the frontal cylinder and the dotted line represents the wave forces on the rear cylinder. Because of the shielding effect of the frontal cylinder the force on the rear cylinder is less than that on the frontal cylinder.

The maximum linear wave force $F_{\max }$ on two tandem cylinders for different separation distance $S / r$, which is the distance between two cylinders, are presented in Fig. 7. Nine cases of the separation distance between the edges of the

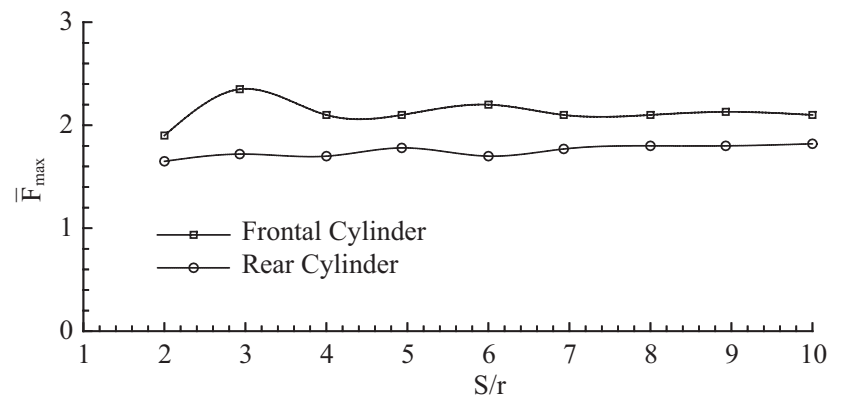

Fig. 7. The computed nondimensional maximum linear wave force versus separation distance $S / r$ for two tandem cylinder array for $k r=$ $2, \lambda=2 \pi$.

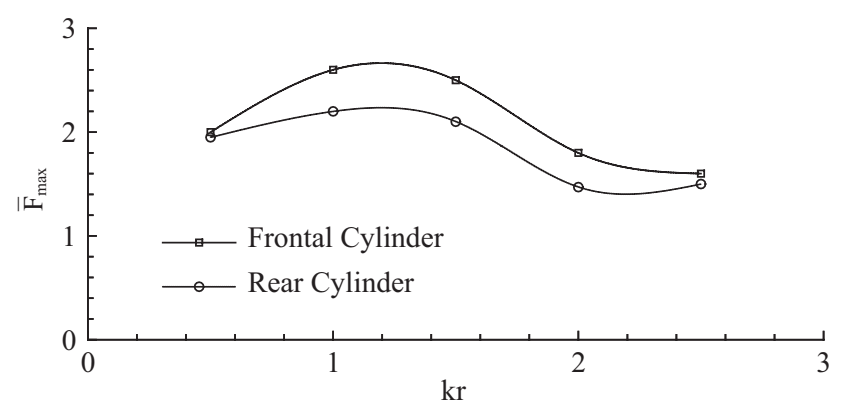

Fig. 8. The computed nondimensional maximum linear wave force versus $k r$ for two tandem cylinder array for $S / r=4, T=2.3, \lambda=2 \pi$.

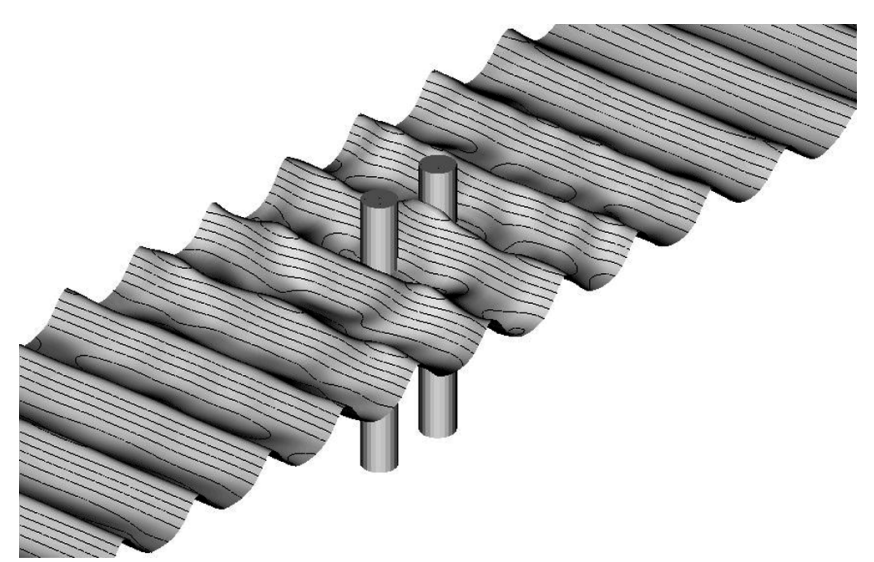

Fig. 9. Perspective view of a fully developed cnoidal wave field at $t=108$ $T$ in presence of two circular cylinders for $\varepsilon=0.3, m=0.90, k r=$ 1.0, $S / r=3, \lambda=12$.

cylinders, ranging from $S=2 r$ to $10 r$ are investigated. The radius of each cylinder is unity, the wave length $\lambda=2 \pi$. The maximum force on the frontal cylinder shows an undulating variation for different $S / r$ values. The frontal cylinder experiences a significant wave force when $S / r=3$. The computed nondimensional maximum linear wave force versus $k r$ for two tandem cylinder array for $S / r=4, T=2.3, \lambda=2 \pi$ is plotted in Fig. 8.

Fig. 9 gives the perspective view of a fully developed cnoidal wave field at $t=108 T$ in presence of two circular 


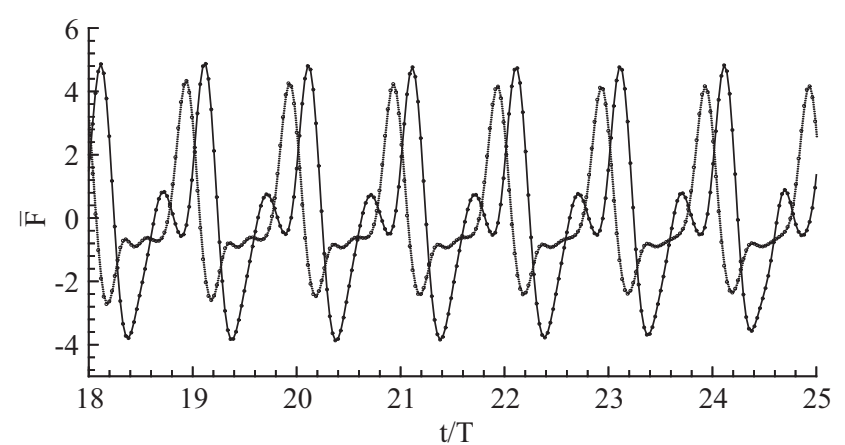

Fig. 10. The computed nondimensional cnoidal wave force on tandem placed circular cylinders for a duration of 7 periods between $t=$ $18 T$ and $t=25 T$ for $\varepsilon=0.3, m=0.90, k r=1, S / r=3, \lambda=12$.

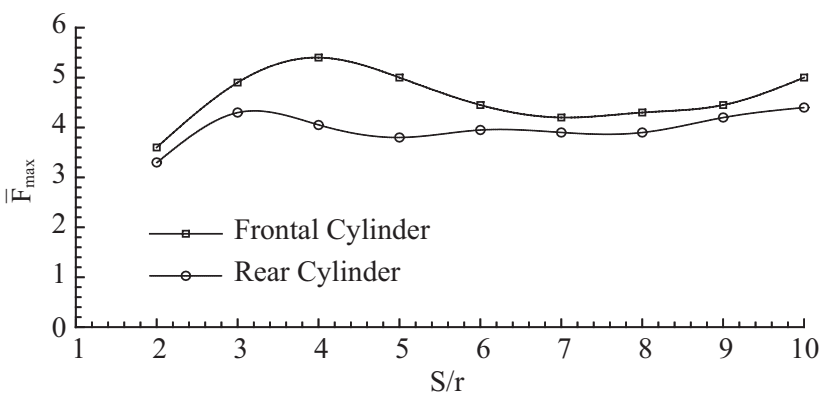

Fig. 11. The computed nondimensional maximum nonlinear wave force versus separation distance $S / r$ for two tandem cylinder array for $\varepsilon=0.3, m=0.90, k r=1, \lambda=12$.

cylinders for $\varepsilon=0.3, m=0.90, k r=1.0, S / r=3, \lambda=12$. Fig. 10 depicts the time variation of the cnoidal wave force on the frontal and rear cylinders for $\varepsilon=0.3, m=0.9$. The solid line represents wave forces on the frontal cylinder and the dotted line represents the wave forces on the rear cylinder. The force on the rear cylinder is again less than that on the frontal cylinder, due to the shielding effect.

The maximum wave force on two tandem cylinders for different separation distance $S / r$ is presented in Fig. 11. Again, nine cases of the separation distance between the edges of the cylinders, ranging from $S=2 r$ to $10 r$ are investigated. The radius of each cylinder is unity, the wave length $\lambda=12$, the $\varepsilon=$ 0.3 . The maximum force on the frontal cylinder shows a periodic variation for different $S / r$ values. The force on the rear cylinder shows less variation than that on the frontal cylinder for different $S / r$ values. The frontal cylinder is subject to a significant wave force when $S / r=4$, the ratio of the maximum force between frontal and rear cylinders takes on a significant value for $3<S / r<6$. The computed nondimensional maximum nonlinear cnoidal wave force versus $k r$ for two tandem cylinder array for $S / r=3, \varepsilon=0.3, m=0.90, k r=1, \lambda=12$ is shown in Fig. 12.

\section{Three Cylinder Array}

As a final case, three cylinders are placed in tandem parallel

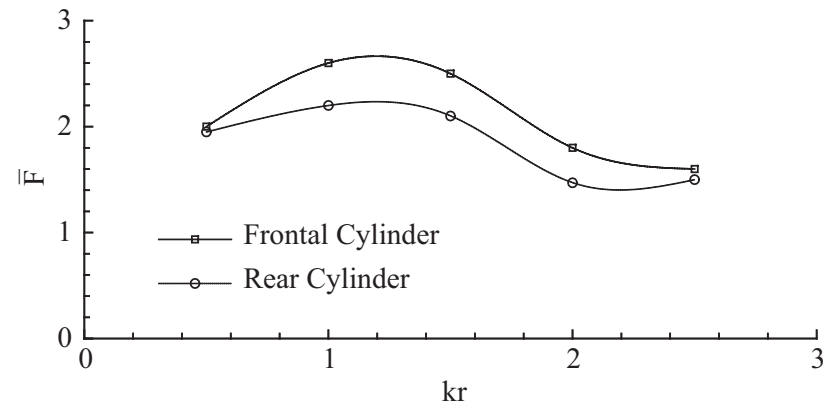

Fig. 12. The computed nondimensional maximum nonlinear cnoidal wave force versus $\mathrm{kr}$ for two tandem cylinder array for $S / r=4$, $\varepsilon=0.3, m=0.90, k r=1, \lambda=12$.

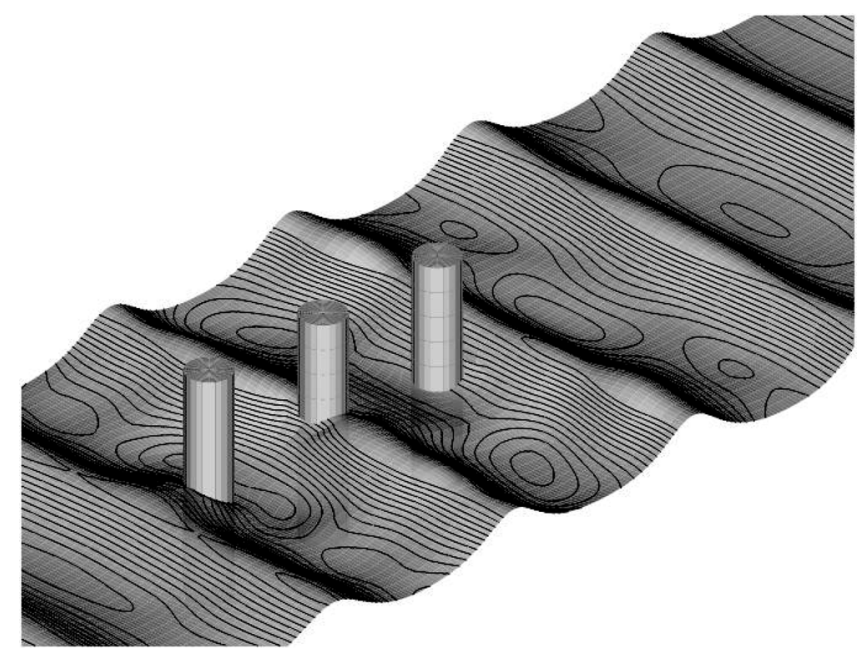

Fig. 13. Three dimensional perspective view of the elevation in presence of the three tandem cylinder array at $t=105 \mathrm{~s}(\mathrm{kr}=1, \varepsilon=0.3, m=$ $0.9, \lambda=6.14 S / r=3$ ).

to the wave direction. The $x$-direction grid size $\Delta x$ ranges from $\lambda / 30$ to $\lambda / 75$ and the $y$-direction grid size $\Delta y$ ranges from $\lambda / 20$ to $\lambda / 50$. The incident wave amplitude is $a_{0}=0.34$, the number of grid points in $x$ - and $y$-directions are 1224 and 40, respectively. The time step $\Delta \tau=0.1(=T / 40) \mathrm{s}$.

Fig. 13 shows a closer three dimensional perspective view of a fully developed wave field in presence of the three tandem placed cylinder array for $k r=1$, after 35 wave periods elapsed. The time variation of the wave force on the frontal, middle and rear cylinders for $\varepsilon=0.3$ is shown in Fig. 14. Again, due to the shielding effect of the frontal cylinder, the force on the rear and middle cylinders is less than that on the frontal one. Time history of the wave force on the three cylinders, Fig. 14, seems all complicated due to both shielding and reflection effects. $F_{\text {max }}$ for the middle cylinder undulates appreciably within the $S / r$ ranges investigated. The force on three tandem cylinders for different separation distance $S / r$ are presented in Fig. 15.

The radius of each cylinder is unity, the wave length $\lambda=2 \pi$, the $\varepsilon=0.3$. The maximum forces on the frontal and rear cylinders show less variation compared to the maximum force 

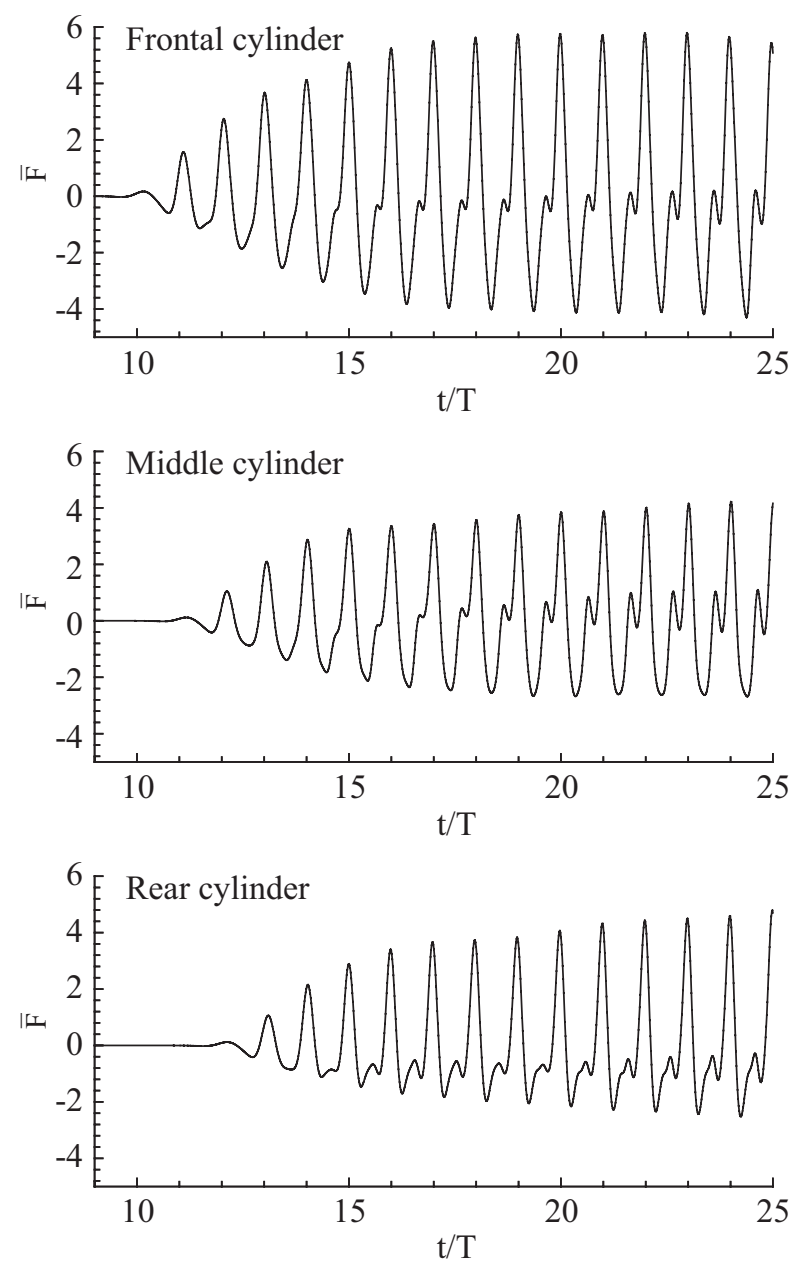

Fig. 14. The computed nondimensional wave force on the frontal, middle and rear cylinders for a duration of 16 wave periods. $k r=1, \varepsilon=$ $0.1, m=0.9, \lambda=12 \mathrm{~m}, T=4.73 \mathrm{~s}, S / r=5$.

on the middle cylinder. The frontal cylinder experiences a significant wave force for $S / r=5$, the middle cylinder for $S / r=$ 3,5 and 8, finally the rear cylinder for $S / r=5,9$, and 10 .

\section{CONCLUSION}

The two-dimensional diffraction of linear and cnoidal waves by a group of tandem placed vertical cylinders and the forces acting on each cylinder are investigated numerically, using a vertically-integrated nonlinear dispersive wave model with insertion of a semi-empirical wave breaking effect, in nonorthogonal curvilinear coordinate system. The use of contravariant velocities provides important numerical advantages. Computations show that the interference between adjacent cylinders has a significant effect on the wave field and forces on each individual cylinder. In accord with the previous works, larger wave forces are obtained for nonlinear cases compared to the linear cases. The wave forces acting on the middle and rear cylinders reduced by the shielding effect. For engineering applications, a series of numerical experiments can be per-

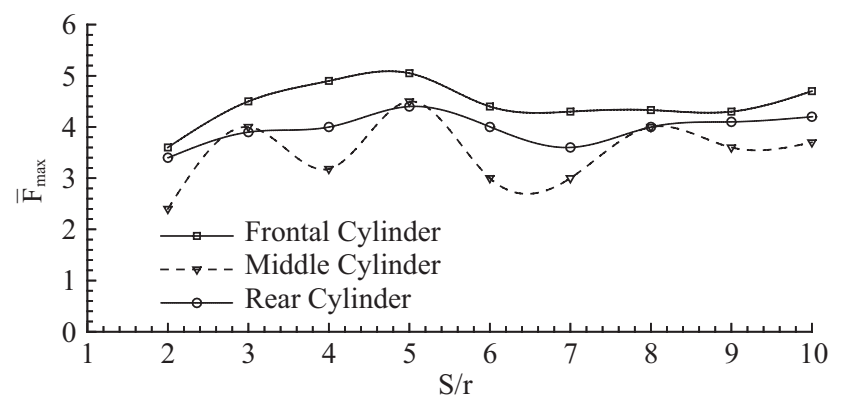

Fig. 15. The computed nondimensional maximum nonlinear wave force versus separation distance $S / r$ for three tandem cylinder array for $\varepsilon=0.3, m=0.90, k r=1, \lambda=12$.

formed for various wave periods and $S / r$ values so that minimum wave forces for a given arrangement of cylinders can be determined. Also, the diameters of the cylinders may be chosen differently to see the effects on wave forces. For the future research, the proposed method can be easily employed to an array of elliptic and arbitrary cross-section cylinders. By adding the current term in the equations, wave-current interaction problems can also be investigated.

\section{ACKNOWLEDGMENTS}

The author would like to thank his colleague Dr. Serdar Beji with his precious discussions.

\section{REFERENCES}

1. Abul-Azm, A. G. and Williams, A. N., "Approximation of second-order diffraction loads on arrays of vertical circular cylinders," Journal of Fluids and Structures, Vol. 3, No. 1, pp. 17-36 (1989).

2. Barlas, B. and Beji, S., "Boundary-fitted numerical model for computing linear and nonlinear wave forces on bottom-mounted piles," Applied Ocean Research, Vol. 28, No. 4, pp. 267-274 (2006).

3. Beji, S. and Barlas, B., "Boundary-fitted non-linear dispersive wave model for regions of arbitrary geometry," International Journal for $\mathrm{Nu}$ merical Methods in Fluids, Vol. 45, No. 6, pp. 643-657 (2004).

4. Beji, S. and Nadaoka, K., "A time-dependent nonlinear mild-slope equation for water waves," Proceedings of the Royal Society London A, Vol. 453, pp. 319-332 (1997).

5. Bidde, D. D., "Laboratory study of lift forces on circular piles," Journal of the Waterways, Harbors and Coastal Engineering, Vol. 97, No. 4, pp. 595-614 (1971).

6. Carstens, B. and Sayer, P. G., "Hydrodynamic interactions between two vertical circular cylinders in linear oscillatory flow," Ocean Engineering, Vol. 24, No. 4, pp. 351-380 (1997).

7. Horikawa, K. and Kuo, C. T., "A study of wave transformation inside surf zone," Proceedings 10th Coastal Engineering Conference, ASCE, New York, Vol. 1, pp. 217-233 (1966).

8. Isaacson, M. and Cheung, K. F., "Time-domain second-order wave diffraction in three dimensions," Journal of Waterway, Port, Coastal and Ocean Engineering ASCE, Vol. 118, No. 5, pp. 496-516 (1992).

9. Kagemoto, H. and Yue, D. K. P., "Interactions among multiple threedimensional bodies in water waves: an exact algebraic method," Journal of Fluid Mechanics, Vol. 166, No. 1, pp. 189-209 (1986).

10. Korteweg, D. J. and de Vries, G., "On the change of form of long waves advancing in a rectangular canal, and on a new type of long stationary waves," Philosophical Magazine, Vol. 39, pp. $422-443$ (1895). 
11. Linton, C. M. and Evans, D. V., "The interaction of waves with a row of circular cylinders," Journal of Fluid Mechanics, Vol. 215, pp. 549-569 (1990).

12. Maniar, H. D. and Newman, J. N. "Wave diffraction by a long array of cylinders," Journal of Fluid Mechanics, Vol. 339, pp. 309-329 (1997).

13. Masuda, K., Kato, W., and Ishizuka, H., "Second-order diffraction load on plural vertical cylinder with arbitrary cross-sections," Proceedings 5th International OMAE Conference, New York, pp. 345-352 (1986).

14. Mavrakos, S. A. and Chatjigeorgiou, I. K., "Second-order diffraction by a bottom-seated compound cylinder," Journal of Fluids and Structures, Vol. 22, No. 4, pp. 463-492 (2006).

15. McIver, P. and Evans, D. V., "Approximation of wave forces on cylinder arrays," Applied Ocean Research, Vol. 6, No. 2, pp. 101-107 (1984).

16. Nadaoka, K., Beji, S., and Nakagawa, Y., "A fully dispersive weakly nonlinear model for water waves," Proceedings of the Royal Society London A, Vol. 453, pp. 303-318 (1997).

17. Ohl, C. O. G., Eatock Taylor, R., and Borthwick, A. G. L., "Water wave diffraction by a cylinder array Part 1. Regular waves," Journal of Fluid Mechanics, Vol. 442, pp. 1-32 (2001).

18. $\mathrm{Su}, \mathrm{M}$. and Pan, Y. "Shallow water diffraction around multiple large cylinders," Applied Ocean Research, Vol. 9, No. 1, pp. 31-36 (1987)

19. Utsunomiya, T. and Eatock Taylor, R., "Trapped modes around a row of circular cylinders in a channel," Journal of Fluid Mechanics, Vol. 386, pp. 259-279 (1999).

20. Walker, D. A. G. and Eatock Taylor, R., "Wave diffraction from linear arrays of cylinders," Ocean Engineering, Vol. 32, No. 17-18, pp. 2053 2078 (2005).

21. Wang, C. Z. and Wu, G. X., "Time domain analysis of second-order wave diffraction by an array of vertical cylinders," Journal of Fluids and Structures, Vol. 23, No. 4, pp. 605-631 (2007).

22. Wang, K. H. and Jiang, L., "Solitary wave interactions with an array of two vertical cylinders," Applied Ocean Research, Vol. 15, No. 6, pp. 337 350 (1994).

23. Wang, K. H. and Ren, X., "Interactions of cnoidal waves with cylinder arrays," Ocean Engineering, Vol. 26, No. 1, pp. 1-20 (1999).

24. Wang, K. H., Wu, T. Y., and Yates, G. T., "Three-dimensional scattering of solitary waves by vertical cylinder," Journal of Waterway, Port, Coastal and Ocean Engineering ASCE, Vol. 118, No. 5, pp. 551-566 (1992).

25. Williams, A. N. and Li, W., "Water wave interaction with an array of bottom-mounted surface-piercing porous cylinders," Ocean Engineering, Vol. 27, No. 8, pp. 841-866 (2000).

26. Yilmaz, O., "An iterative procedure for the diffraction of water waves by multiple cylinders," Ocean Engineering, Vol. 31, No. 11-12, pp. 14371446 (2004). 